\title{
On the Weak Convergence of Stochastic Processes without Discontinuities of the Second Kind
}

\author{
MichaEL WoODRoofe
}

Received January 29, 1968

\begin{abstract}
Summary. Subspaces $D_{\alpha}, \alpha>0$, of $D[0,1]$ are defined and given complete metries $d_{\kappa}$ which are stronger than the Prokhorov metric. The spaces $\left(D_{\alpha}, d_{\alpha}\right)$ are shown to be separable, and their pre-compact subsets are characterized. A condition which is known to guarantee weak pre-compactness of sets of probability measures over $D[0,1]$ is shown to also guarantee weak pre-compactness of probability measures over $D_{\alpha}$ for appropriate values of $\alpha$. Applications are made to the weak convergence of measures induced by stochastic processes, and some examples are included.
\end{abstract}

\section{Introduction}

The spaces $C[0,1]$ and $D[0,1]$ have been the setting for much of the recent work on the weak convergence of stochastic processes, and several criteria which guarantee the weak precompactness of sets of measures in $C[0,1]$ and $D[0,1]$ are now known. LAMPERTI [3] observed that one of these criteria, due essentially to KoLMogorov $([4]$, p. 519), actually guarantees weak precompactness with respect to the topology of $\operatorname{Lip}_{\alpha}[0,1]$ for appropriate values of $\alpha$. The transition from $C[0,1]$ to $\operatorname{Lip}_{\alpha}[0,1]$ is desirable because, as explained in [3], it enlarges the class of continuous functionals.

Here we obtain an improvement of theorems on the weak convergence of stochastic processes in $D[0,1]$ which is analogous to that given by LAMPERTx for $C[0,1]$. To do so we need analogoues of (1) KoLmogonov's criterion and (2) the spaces $\operatorname{Lip}_{\alpha}[0,1]$. The former has been given by Chentsov [1]. The latter, which we have denoted by $D_{\alpha}$, are discussed in section two and are to the best of our knowledge new. In section three we show that (a slightly modified version of) the Chentsov criterion does, indeed, guarantee weak precompactness with respect to the topology of $D_{\alpha}$. And in section four we give examples of stochastic processes and functionals to which our results apply.

\section{The Spaces $D_{\alpha}$}

By $(D, d)$ we will understand the complete, separable metric space which is constructed in Chapter VII of [5] and is a variation on the space $D[0,1]$ of [6]. Thus the elements of $D$ are right continuous, real valued functions which are defined on $[0,1]$, have no discontinuities of the second kind, and are continuous at zero and one. The metric $d$ is defined by

$$
d(f, g)=p\left(\Gamma_{f}, \Gamma_{g}\right)+L\left(m_{f}, m_{g}\right),
$$

where $p\left(\Gamma_{f}, \Gamma_{g}\right)$ denotes the divergenee between the graphs of $f$ and $g([5],[6])$, 
$L$ denotes the Lévy metric for monotone functions on the line ([2], pp. 38, 39), and $m_{f}$ is defined as follows: for $0<\delta \leqq 1$ let $\bar{\omega}_{f}(\delta)$ be the maximum of

and

$$
\sup _{0 \leqq t \leqq \delta}|f(t)-f(0)|, \quad \sup _{1-\delta \leqq t \leqq 1}|f(t)-f(1)|,
$$

$$
\hat{\omega}_{f}(\delta)=\sup \min \left\{\left|f\left(t_{1}\right)-f\left(t_{0}\right)\right|,\left|f\left(t_{0}\right)-f\left(t_{2}\right)\right|\right\},
$$

where the third supremum is taken over $t_{1}, t_{0}, t_{2} \in[0,1]$ for which

$$
t_{0}-\delta \leqq t_{1} \leqq t_{0} \leqq t_{2} \leqq t_{0}+\delta \text {; }
$$

then $m_{f}(z)=\bar{\omega}_{f}\left(e^{z}+0\right)$ for $z \leqq 0$ and $m_{f}(z)=m_{f}(0)$ for $z \geqq 0$. For $\alpha>0$ we define $D_{\alpha}$ to be the set of $f \in D$ for which

$$
\int_{-\infty}^{\infty} m_{f}(z) e^{-\alpha z} d z<\infty
$$

and we topologize $D_{\alpha}$ with the metric $d_{\alpha}$ defined by

$$
\begin{aligned}
d_{\alpha}(f, g) & =p\left(\Gamma_{f}, \Gamma_{g}\right)+L_{\alpha}\left(m_{f}, m_{g}\right), \\
L_{\alpha}\left(m_{f}, m_{g}\right) & =\int_{-\infty}^{\infty}\left|m_{f}(z)-m_{g}(z)\right| e^{-\alpha z} d z .
\end{aligned}
$$

$d_{\alpha}$ is a metric because both $p$ and $L_{\alpha}$ are and because $f=g$ if and only if $\Gamma_{f}=\Gamma_{g}$. Moreover, $d_{\alpha}$ is stronger than $d$ because $L_{\alpha}$ is stronger than $L$.

Our immediate goal is to ascertain some of the topological properties of the spaces $\left(D_{\alpha}, d_{\alpha}\right)$, which we will denote simply by $D_{\alpha}$ in the following. They resemble and to some extent follow from those of $(D, d)$, which we will denote by $D$ in the following.

Theorem 2.1. $A$ subset $F$ of $D_{\alpha}$ is precompact in $D_{\alpha}$ if and only if

$$
\begin{gathered}
\sup _{f \in F^{t}} \sup _{0 \leqq t \leqq 1}|f(t)|<\infty, \\
\lim _{a \rightarrow-\infty} \sup _{f \in F} \int_{-\infty}^{a} m_{f}(z) e^{-\alpha z} d z=0 .
\end{gathered}
$$

Proof. (Sufficiency.) Let $F$ satisfy (2.1) and (2.2); then the inequality,

$$
\bar{\omega}_{f}(\delta) \leqq e \delta^{\alpha} \int_{-\infty}^{(\log \delta)+1} m_{f}(z) e^{-\alpha z} d z, \quad 0<\delta<e^{-1},
$$

shows that $\bar{\omega}_{f}(\delta) \rightarrow 0$ as $\delta \rightarrow 0$ uniformly in $f \in F$, so that $F$ is precompact in $D$ ([5], Ch. VII, Thm. 6.2). Therefore, if $f_{n} \in F, n \geqq 1$, there exists a subsequence $n_{k}$, $k \geqq 1$, and an $f_{0} \in D$ for which $d\left(f_{n_{k}}, f_{0}\right) \rightarrow 0$ as $k \rightarrow \infty$. Moreover, $f_{0} \in D_{\alpha}$ by FAToU's Lemma, (2.1) and (2.2). Now the convergence of $f_{n_{k}}$ to $f_{0}$ in $D$ implies the convergence of $m_{f_{k}}(z)$ to $m_{f_{0}}(z)$ for all $z$ at which $m_{f_{0}}$ is continuous and, in particular, almost everywhere with respect to Lebesgue measure. (2.2) now implies $d_{\alpha}\left(f_{n_{k}}, f_{0}\right) \rightarrow 0$ as $k \rightarrow \infty$. The sufficiency follows. 
(Necessity.) If $F$ is precompact in $D_{\alpha}$, then, since $d_{\alpha}$ is stronger than $d, F$ is precompact in $D$ so that (2.1) follows from the theorem cited above. If (2.2) were false, then there would exist sequences $a_{n} \in R^{1}$ and $f_{n} \in F$ for which $a_{n} \rightarrow-\infty$ as $n \rightarrow \infty$ and

$$
\liminf _{n \rightarrow \infty} \int_{-\infty}^{a_{n}} m_{f_{n}}(z) e^{-\alpha z} d z>0 .
$$

There would also be a subsequence $n_{k}, k \geqq 1$, and an $f_{0} \in D_{\alpha}$ for which $d_{\alpha}\left(f_{n_{k}}, f_{0}\right) \rightarrow 0$ as $k \rightarrow \infty$ and, in particular,

$$
\lim _{k \rightarrow \infty} \int_{-\infty}^{\infty}\left|m_{f_{n_{k}}}(z)-m_{f_{0}}(z)\right| e^{-\alpha z} d z=0 .
$$

The necessity of (2.2) follows from the incompatibility of (2.4) and (2.5). Q.E.D.

Corollary 2.1. $D_{\alpha}$ is complete.

Corollary 2.2. Let $f_{n} \in D_{\alpha}, n \geqq 0$; sufficient conditions for $d_{\alpha}\left(f_{n}, f_{0}\right) \rightarrow 0$ as $n \rightarrow \infty$ are that (1) $f_{n}(t) \rightarrow f_{0}(t)$ for all $t$ belonging to a dense subset of $(0,1)$; and (2) that $m_{f_{n}}(\cdot) e^{-\alpha(\cdot)}$ be dominated by an integrable function on $(-\infty, 0]$.

Proof. Corollary 2.1 follows from the easily verified fact that a Cauchy sequence satisfies (2.1) and (2.2). To establish Corollary 2.2 we need only observe that $f_{n}$ is precompact and that if $f$ is any limit point of $f_{n}$, then $p\left(\Gamma_{f}, \Gamma_{f_{0}}\right)=0$ so that $f=f_{0}$.

Q.E.D.

Theorem 2.2. $D_{\alpha}$ is separable.

Proof. Let $D^{n}$ be the set of those $f \in D_{\alpha}$ which take a constant, rational value on each of the intervals $[(i-1) / n, i / n), i=1, \ldots, n \geqq 1$ and let $D^{0}=\bigcup_{n=1}^{\infty} D^{n}$. If $f \in D_{\alpha}$ and $t_{1}^{n}, \ldots, t_{n}^{n}$ are continuity points of $f$ for which $i-1<n t_{i}^{n}<i$, $i=i, \ldots, n$, then there is an $f_{n} \in D^{n}$ which differs from $f$ by less than $n^{-2 \alpha}$ at each of the points $t_{i}^{n}$. It follows easily that $f_{n}(t) \rightarrow f(t)$ as $n \rightarrow \infty$ at all continuity points $t$ of $t$, the totality of which are dense in $(0,1)$. Moreover, we have $\bar{\omega}_{f_{n}}(\delta) \leqq \bar{\omega}_{f}(\delta+(2 / n))+2 n^{-2 \alpha}, 0<\delta \leqq 1-(2 / n)$.

For example,

$$
\begin{aligned}
\sup _{0 \leqq t \leqq \delta}\left|f_{n}(t)-f_{n}(0)\right| & =\max _{1 \leqq i \leqq k}\left|f_{n}\left(t_{i}^{n}\right)-f_{n}(0)\right| \\
& \leqq \max _{1 \leqq i \leqq k}\left|f\left(t_{i}^{n}\right)-f(0)\right|+2 n^{-2 \alpha} \\
& \leqq \sup _{0 \leqq t \leqq \delta+(1 / n)}|f(t)-f(0)|+2 n^{-2 \alpha}
\end{aligned}
$$

where $k$ is one plus the greatest integer in $n \delta$. Since $\bar{\omega}_{f_{n}}(\delta)=0$ for $0<\delta<(1 / n)$, Corollary 2.2 applies to show that $d_{\alpha}\left(f_{n}, f\right) \rightarrow 0$ as $n \rightarrow \infty$; and since $D^{0}$ is countable, the theorem follows.

Q.E.D.

We conclude this section with a discussion of the subspace $C_{\alpha}=D_{\alpha} \cap C[0,1]$. The inequality ([5], p. 235)

$$
\omega_{f}(\delta) \leqq 2 \bar{\omega}_{f}(\delta)+\eta(f)
$$

where $\omega_{f}$ denotes the modulus of continuity and $\eta(f)$ the maximal discontinuity of $f$ shows (1) that $C_{\alpha} \subset \operatorname{Lip} \alpha[0,1] ;(2)$ that $C_{\alpha}$ is closed in $D_{\alpha}$ (Ascorr's Theorem); 
and (3) that the identity mapping of $C_{\alpha}$ into $\operatorname{Lip}_{\alpha}[0,1]$ is continuous. In the other direction we may only conclude that if $f \in \operatorname{Lip}_{\alpha}[0,1]$, then $f \in C_{\beta}$ for all $\beta<\alpha$ and that the identity mapping from $\operatorname{Lip}_{\alpha}[0,1]$ into $C_{\beta}$ is continuous.

\section{Measures in $D_{\alpha}$}

By a measure in $D_{\alpha}$ we will mean a probability measure defined on the Borel sets $B_{\alpha}$ of $D_{\alpha}$, and we will denote the space of all measures in $D_{\alpha}$ by $M_{\alpha}$. The topology of $M_{\alpha}$ is the weak topology it inherits as a subset of $C\left(D_{\alpha}\right)^{*}$, the dual space to the space of continuous functionals on $D_{\alpha}$. The following lemma with $D_{\alpha}$ replaced by $D$ is proved in [5], p. 251 ; the proof given there extends to $D_{\alpha}$ in a straightforward manner.

Lemma 3.1. $B_{\alpha}$ is the smallest $\sigma$-algebra with respect to which the functionals, $\pi_{t}: f \rightarrow f(t), 0 \leqq t \leqq 1$, are measurable.

If $X(t), 0 \leqq t \leqq 1$, is a stochastic process, and $\mu \in M_{\alpha}$, we will say that $\mu$ has been induced by $X(t), 0 \leqq t \leqq 1$, if and only if the following equality holds for every finite subset $T$ of $[0,1]$ and every choice of $x_{t} \in R^{1}, t \in T$ :

$$
\mu\left(\left\{f \in D_{\alpha}: f(t) \leqq x_{t}, t \in T\right\}\right)=\operatorname{Pr}\left(X(t) \leqq x_{t}, t \in T\right) .
$$

By Lemma 3.1 a stochastic process can induce at most one measure.

Theorem 3.1. Let $X(t), 0 \leqq t \leqq 1$, be a stochastically right continuous stochastic process. If there exist monotone functions $q_{i}:(0,1] \rightarrow[0, \infty)$ for which $q_{i}(h) \rightarrow 0$ as $h \rightarrow 0, i=1,2,3, \sum_{k=1}^{\infty} q_{i}\left(2^{-k}\right)<\infty i=1,2$, and

$$
\begin{aligned}
& \operatorname{Pr}\left(\left|X\left(t_{i+1}\right)-X\left(t_{i}\right)\right| \geqq h^{\alpha} q_{1}(h), i=1,2\right) \leqq h q_{2}(h), \\
& \quad \operatorname{Pr}\left(\left|X(i)-X\left(i+h(-1)^{i}\right)\right| \geqq h^{\alpha} q_{1}(h)\right) \leqq q_{3}(h), \quad i=0,1,
\end{aligned}
$$

whenever $0 \leqq t_{1} \leqq t_{2} \leqq t_{3} \leqq 1, t_{3}-t_{1} \leqq h$, and $0<h \leqq 1$, then $X(t), 0 \leqq t \leqq 1$, induces a measure in $D_{\alpha}$.

Proof. Define stochastic processes $X_{k}(t), 0 \leqq t \leqq 1, k \geqq 1$, by

$$
X_{k}(t)=\sum_{i=1}^{2^{k}} X\left(i 2^{-k}\right) I\left(2^{k}, i ; t\right),
$$

where $I(n, i ; \cdot)$ is the indicator function of $[i-1) / n, i / n), i=1, \ldots, n-1$, and $I(n, n ; \cdot)$ is the indicator function of $[(n-1) / n, 1] ;$ then each $X_{k}(t), 0 \leqq t \leqq 1$, induces a measure $\mu_{k}$ in $D_{\alpha}$. We will show first that the sequence $\mu_{k}$ is precompact. Let $B_{k, i}^{c}$ be the set of $f \in D_{\alpha}$ for which

$$
\left|f\left((j-1) 2^{-k}\right)-f\left(j 2^{-k}\right)\right| \geqq q_{1}\left(2^{-k}\right) 2^{-\alpha k}
$$

for $j=i, i+1$ if $1 \leqq i<2^{k}$, for $j=1$ if $i=0$, and for $j=2^{k}$ if $i=2^{k}$, and let $E_{n}=\bigcap_{k=n}^{\infty} \bigcap_{i=0}^{2^{k}} B_{k, i}$. Then

$$
\begin{gathered}
E_{n} \subset\left\{f \in D_{\alpha}: \bar{\omega}_{f}\left(2^{-p}\right) \leqq q\left(2^{-p}\right) 2^{-\alpha p}, p \geqq n\right\}=F_{n} \quad \text { say, } \\
\mu_{k}\left(E_{n}^{c}\right) \leqq 2\left(\sum_{p=n}^{\infty} q_{2}\left(2^{-p}\right)+q_{3}\left(2^{-n}\right)\right), \quad k \geqq 1,
\end{gathered}
$$


for $n \geqq 1$ where $q(h)=16\left(1-2^{-\alpha}\right)^{-1} q_{1}(2 h), \quad 0<h \leqq(1 / 2)$. That $\hat{\omega}_{f}\left(2^{-p}\right)$ $\leqq q\left(2^{-p}\right) 2^{-\alpha p-1}, p \geqq n$, for $f \in E_{n}$ is essentially shown in [1], Lemmas 1 and 2; moreover, $|f(\delta)-f(0)| \leqq w$ and $\hat{\omega}_{f}(\delta) \leqq w$ imply $\sup _{0 \leqq t \leqq \delta}|f(t)-f(0)| \leqq 2 w$. Next let $G_{b}$ be the set of $f \in D_{\alpha}$ which are bounded by $2 b$; then for $b>q((1 / 2))$ and $n \geqq 1$ we have

$$
\mu_{k}\left(G_{b}^{c}\right) \leqq \operatorname{Pr}\left(\max _{0 \leqq i \leqq 2^{n}}\left|X\left(i 2^{-n}\right)\right| \geqq b\right)+\mu_{k}\left(E_{n}^{c}\right), \quad k \geqq 1,
$$

which may be made arbitrarily small by taking $n$ and then $b$ sufficiently large. Since $F_{n} \cap G_{b}$ is compact for every $n$ and $b$, it follows from (3.3) and (3.4) that $\mu_{k}, k \geqq 1$, is precompact.

Now we claim that any limit point of $\mu_{k}, k \geqq 1$, must satisfy $(3.1)-$ i.e. that $\mu_{k}$ converges to a measure induced by $X(t), 0 \leqq t \leqq 1$. We may assume that $X(t)$, $0 \leqq t \leqq 1$, is separable and that $T \subset S$, the set of diadic rationals of $(0,1)$. Let $\mu$ be a limit point of $\mu_{k}, k \geqq 1$, let $T$ be a finite subset of $S$, and for each $t \in T$ let $h_{n, t}$ be a continuous function for which $0 \leqq h_{n, t} \leqq 1, h_{n, t}(s)=0$ if either $s \leqq t$ or $s \geqq t+2^{-n+1}$, and $h_{n, t}\left(t+2^{-n}\right)=1$. Then, since the functionals

$$
f \rightarrow \sup _{0 \leqq s \leqq 1} h_{n, t}(s) f(s)
$$

are continuous on $D_{\alpha}$ for every $n \geqq 1$ and $t \in T([5]$, p. 249), we must have

$$
\mu\left(\left\{f: \sup _{0 \leqq s \leqq 1} h_{n, t}(s) f(s) \leqq x_{t}, t \in T\right\}\right)=\operatorname{Pr}\left(\sup _{s \in S} h_{n, t}(s) X(s) \leqq x_{t}, t \in T\right)
$$

for every choice of $x_{t} \in R^{1}, t \in T$. When $n \rightarrow \infty,(3.1)$ follows from (3.3b) and the stochastic right continuity of $X(t), 0 \leqq t \leqq 1$.

Q.E.D.

The next theorem is a straightforward generalization of Theorem 3.1, the key observation being that $\mu\left(F_{n}^{c}\right) \leqq \liminf _{k \rightarrow \infty} \mu_{k}\left(F_{n}^{c}\right)$ where $\mu$ and $\mu_{k}$ are as in Theorem 3.1.

Theorem 3.2. Let $X_{n}(t), 0 \leqq t \leqq 1, n \geqq 0$, be a sequence of stochastically right continuous stochastic processes, each of which satisfies (3.2) with $q_{1}, q_{2}$, and $q_{3}$ independent of $n$. Also let $\mu_{n}$ be the measure induced by $X_{n}(t), 0 \leqq t \leqq 1, n \geqq 0$. $A$ sufficient condition for $\mu_{n}, n \geqq 1$, to be precompact (converge to $\mu_{0}$ ) is that for every finite subset $T=\left\{t_{1}, \ldots, t_{k}\right\} \subset[0,1]$ the family of joint distributions of $\left(X_{n}\left(t_{1}\right), \ldots, X_{n}\left(t_{k}\right)\right), n \geqq 1$, be precompact (converge to the joint distribution of $\left.\left(X_{0}\left(t_{1}\right), \ldots, X_{0}\left(t_{k}\right)\right)\right)$.

We conclude this section with a lemma which gives sufficient conditions for (3.2) to hold. It is an easy consequence of Markov's Inequality.

Lemma 3.2. If there are positive constants $K, b, b_{1}, b_{2}, \beta$, and $\gamma$ for which

$$
\begin{gathered}
E\left(\left|X_{n}\left(t_{1}\right)-X_{n}\left(t_{2}\right)\right|^{b_{1}}\left|X_{n}\left(t_{2}\right)-X_{n}\left(t_{3}\right)\right|^{b_{2}}\right) \leqq K h^{1+\beta}, \\
E\left(\left|X_{n}(i)-X_{n}\left(i+h(-1)^{i}\right)\right|^{b}\right) \leqq K h \nu, \quad i=0,1,
\end{gathered}
$$

whenever $0 \leqq t_{1} \leqq t_{2} \leqq t_{3} \leqq 1, t_{3}-t_{1} \leqq h$, and $n \geqq 0$, then for any

$$
\alpha<\min \left\{\beta /\left(b_{1}+b_{2}\right), \gamma / b\right\}
$$


the processes $X_{n}(t), 0 \leqq t \leqq 1, n \geqq 0$, satisfy (3.2) with $q_{1}(h)=h^{\delta}$ and $q_{2}(h)$ $=q_{3}(h)=K h^{\varepsilon}$ for appropriate $\varepsilon, \delta>0$.

\section{Examples}

Example 1. (The sample distribution function.) Let $X_{1}, X_{2}, \ldots$ be independent, identically distributed random variables whose common distribution function $F$ has a bounded density which vanishes off $[0,1]$. Define

$$
X_{n}(t)=\sqrt{n}\left(F_{n}(t)-F(t)\right), \quad 0 \leqq t \leqq 1, \quad n \geqq 1,
$$

where $F_{n}$ denotes the sample distribution function of $X_{1}, \ldots, X_{n}$. Then the following is true (cf. [1] and [5], pp. 259-267): (1) the finite dimensional distributions of $X_{n}(t), 0 \leqq t \leqq 1$, converge to those of a normal process $X_{0}(t), 0 \leqq t \leqq 1$, with covariance function $K(s, t)=F(s)(1-F(t)), 0 \leqq s \leqq t \leqq 1$; and (2) (3.5) is satisfied with $b=b_{1}=b_{2}=2$ and $\beta=\gamma=1$. It follows from Theorem 3.2 that the measures induced in $D_{\alpha}$ by $X_{n}(t), 0 \leqq t \leqq 1$, converge to that induced by $X_{0}(t), 0 \leqq t \leqq 1$, for $0<\alpha<(1 / 4)$.

Example 2. (Convergence to an infinitely divisible law: bounded variances case.) Let $X_{n, 1}, \ldots, X_{n, k_{n}}, n \geqq 1$, be a triangular array of random variables which are independent and identically distributed in each row. Suppose that the distribution function and variance of $S_{n, k_{n}}=\sum_{k=1}^{k_{n}} X_{k}$ converge to those of an infinitely divisible law (with finite, positive variance). It follows from the theory presented in [2] that the finite dimensional distributions of

$$
X_{n}(t)=\sum_{k=1}^{k_{n}} S_{n, k} I\left(k_{n}, k ; t\right), \quad 0 \leqq t \leqq 1, n \geqq 1,
$$

converge as $n \rightarrow \infty$ to those of an infinitely divisible process, $X_{0}(t), 0 \leqq t \leqq 1$. Moreover, if $0 \leqq t_{1} \leqq t_{2} \leqq t_{3} \leqq 1,\left|t_{3}-t_{1}\right| \leqq h$, and $n \geqq 0$ then

$$
E\left(\prod_{i=1}^{2}\left(X_{n}\left(t_{i}\right)-X\left(t_{i+1}\right)\right)\right)^{2} \leqq K\left(h \sigma^{2}+4(h \Theta)^{2}\right)^{2}
$$

where $\Theta$ and $\sigma^{2}$ are respectively the mean and variance of the limit law and $K$ is independent of $n$. Thus (3.5a) is satisfied with $b_{1}=b_{2}=2$ and $\beta=1$, and, similarly, we may verify that $(3.5 \mathrm{~b})$ is satisfied with $b=2$ and $\gamma=1$. Therefore, by Theorem 3.2 the measures induced by $X_{n}(t), 0 \leqq t \leqq 1$, converge in $D_{\alpha}$ to that induced by $X_{0}(t), 0 \leqq t \leqq 1$, for $0<\alpha<(1 / 4)$.

Example 3. (Convergence to a stable law.) Let $X_{1}, X_{2}, \ldots$ be independent, identically distributed random variables whose common distribution function $F$ belongs to the domain of normal attraction of a stable law of characteristic exponent $\gamma, 0<\gamma<2$. For the sake of convenience, we suppose also that $F$ is

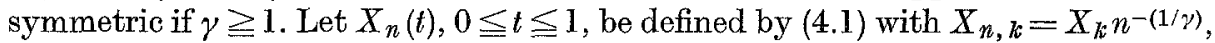
$k=1, \ldots, n$; then, as above, the finite dimensional distributions of $X_{n}(t)$, $0 \leqq t \leqq 1$, will converge to those of a stable process, $X_{0}(t), 0 \leqq t \leqq 1$. We show that (3.2) is satisfied for any $\alpha, 0<\alpha<(1 / 2 \gamma)$. Indeed, since $x^{\gamma}(1-F(x)+F(-x))$ 
is convergent as $x \rightarrow \infty$ ([2], p. 182) and therefore bounded, we have by a standard argument that

$$
\sup _{t \geq 0} t^{-\gamma}|\varphi(t)-1| \leqq B
$$

where $\varphi$ is the characteristic function of $X_{1}$. The inequality, $\left|z^{k}-1\right| \leqq k|z-1|$, $|z| \leqq 1$, now shows that (4.2) also holds when $\varphi$ is replaced by $\varphi_{k}$, the characteristic function of $k^{-(1 / \gamma)} \sum_{i=1}^{k} X_{i}$; and from the truncation inequality ([4], p. 196) we may now infer that

$$
\left.x^{\gamma} \operatorname{Pr}\left(\left|k^{-(1 / \gamma)} \sum_{i=1}^{k} X_{i}\right|\right) \geqq x\right) \leqq B_{1}
$$

is uniformly bounded in $x \geqq 0$ and $k \geqq 1$. (3.2) is an easy consequence: for example

$$
\operatorname{Pr}\left(\left|X_{n}(t)-X_{n}(0)\right| \geqq t^{\alpha}\right) \leqq B_{1} t^{1-\alpha \nu}, \quad 0 \leqq t \leqq 1, n \geqq 1 .
$$

A similar analysis will show that the limit process also satisfies (3.2) for any $\alpha<1 / 2 \gamma$ so that Theorem 3.2 applies.

Examples of continuous functionals on $D_{\alpha}$ which are not continuous on all of $D$ are easy to construct. In particular, any functional of the form.

$$
\Phi(f)=\int_{-\infty}^{0} \varphi(z) m_{f}(z) e^{-\alpha z} d z, \quad f \in D_{\alpha},
$$

where $\varphi$ is a bounded measurable function on $(-\infty, 0]$ are continuous on $D_{\alpha}$ and not even defined on all of $D$. Such functionals might be of interest if, for example, one thinks of $\bar{\omega}_{f}$ as measuring the smoothness of $f$. Another class of functionals to which our results apply is illustrated by

Example 4. (The sample density.) Let $F_{n}$ and $X_{n}(t), 0 \leqq t \leqq 1, n \geqq 1$, be as in Example 1 and define

$$
f_{n}(t)=\left(F_{n}\left(t+h_{n}\right)-F_{n}\left(t-h_{n}\right)\right) / 2 h_{n}, \quad 0 \leqq t \leqq 1, n \geqq 1,
$$

where $0<h_{n} \rightarrow 0$ with $n h_{n}^{2(1-\alpha)} \rightarrow \infty, \alpha<\frac{1}{4} . f_{n}$ provides an estimate of the density $f$ of $X_{1}, X_{2}, \ldots$ (See [7] and the references given there.) Now

$$
\sup _{0 \leqq t \leqq 1}\left|f_{n}(t)-E\left(f_{n}(t)\right)\right|=\sup _{0 \leqq t \leqq 1}\left|X_{n}\left(t+h_{n}\right)-X_{n}\left(t-h_{n}\right)\right| / 2 h_{n} \sqrt{n}
$$

where $X_{n}(t)=0$ for $t<0$ and $t>1$. It follows easily from (2.6) and Example 1 that the right side of (4.3) converges to zero in probability as $n \rightarrow \infty$. If $f$ is continuous, then we also have $E\left(f_{n}(t)\right) \rightarrow f(t)$ uniformly in $t$ ([7]) so that $f_{n}$ will be uniformly consistent. While stronger results on the uniform consistency of sample densities are available ([7] and the references given there), this is apparently the first which makes use of the theory of weak convergence of stochastic processes.

\section{References}

1. Chentsov, N. N.: Weak convergence of stochastic processes whose trajectories have no discontinuities of the second kind and the "heuristic approach to the KolmogorovSmirnov tests". Theor. Probab. Appl. 1, 140-144 (1956).

2. Kotmogonov, A. N., and B. V. GNmbenko: Limit theorems for sums of independent random variables. Cambridge: Addison-Wesley 1954. 
3. LAMPERTi, J.: On the convergence of stochastic processes. Trans. Amer. math. Soc. 104, $430-435$ (1962).

4. Lo亡̀ve, M.: Probability theory. Princeton, N.Y.: Van Nostrand 1962.

5. Parthasarathy, K. R.: Probability measures on metrio spaces. New York: Academic Press 1967.

6. Prokнorov, $\mathrm{Yu}$. V.: Convergence of random processes and limit theorems in probability. Theor. Probab. Appl. 1, $157-214$ (1956).

7. WoonRoofe, M.: On the maximum deviation of the sample density. Ann. math. Statistics $38,475-481$ (1967).

\author{
Dr. Mrohate Woodroofe \\ Mathematics Department \\ University of Michigan \\ Ann Arbor, Michigan 48104, USA
}

